\title{
ARTIGOS
}

\section{BRAND EQUITY: COMPORTAMENTO DO CONSUMIDOR PERANTE AS MARCAS DA DISTRIBUIÇÃO}

\section{Daniela Alexandra da Costa Sousa danielasousa92@gmail.com Mestre em Marketing pela Faculdade de Economia da Universidade de Coimbra- Portugal.}

\section{Arnaldo Coelho} coelho1963@hotmail.com PHD em Gestão. Professor e coordenador dos cursos de doutoramento e MBA da Universidade de Coimbra Portugal.

Cristela Maia Bairrada cristela.bairrada@ua.pt Doutora em Gestão. Professora na Faculdade de Economia da Universidade de Coimbra e no Instituto Superior de Contabilidade e Administração da Universidade de Aveiro Portugal.

\section{RESUMO}

O tema do brand equity não só é relevante para as organizações como também oferece um conjunto vasto de vantagens para o próprio consumidor. O objetivo central desta pesquisa passa por estudar as variáveis que mais contribuem para a sua formação e, ao mesmo tempo, estudar o impacto do brand equity na intenção de compra e no word-of-mouth (WOM). Para os devidos efeitos, foi realizada uma pesquisa de índole quantitativa por meio de um questionário estruturado, baseado em uma amostra de 237 inquiridos com idade superior a 18 anos. Feita a análise estatística, as variáveis price deals, identificação com a marca, confiança na marca foram as que apresentaram um impacto positivo total nas variáveis respeitantes à construção do brand equity: qualidade percebida, lealdade e brand awareness. Já estas dimensões do brand equity parecem ter um impacto positivo no word-of-mouth e a dimensão brand awareness tem um impacto positivo na intenção de compra. Por fim, a intenção de compra tem um impacto positivo no WOM.

Palavras-chave: Brand Equity. Marcas do Distribuidor. Comportamento do Consumidor.

\section{INTRODUÇÃO}

O brand equity é um tópico cada vez mais abordado pelos profissionais de marketing e investigadores. No entanto, a abordagem ao brand equity tem sido feita maioritariamente em relação a marcas que são alvo de grande notoriedade e reconhecimento por parte do consumidor. Assim, torna-se relevante investigar o comportamento do consumidor em relação às marcas da distribuição, isto é, saber o que o consumidor sente em relação a este tipo de marcas e qual o motivo que os leva a adquirir os seus produtos. Nos últimos anos, as marcas de distribuidor têm evidenciado um crescimento significativo na quota de mercado (LAMEY et al., 2007). Em determinadas categorias de produto, já podemos verificar que a marca dominante é a marca do distribuidor(DICK; JAIN; RICHARDSON, 1995; BALTAS, 1997; KELLER, 1998). Segundo Binninger (2008), os consumidores encaram os produtos da marca do distribuidor como uma boa compra uma vez que 
são marcas caracterizadas por sua elevada disponibilidade ao nível da distribuição de lojas.

Existem várias perspetivas acerca do conceito de Brand Equity. Segundo Farquhar (1989), o termo dito de valor da marca refere-se ao valor incremental adicionado pelo nome da marca para um produto (SPRY; PAPPU; CORNWELL, 2011). Para Keller (2003), a gestão do valor da marca significa um grande controlo dos significados que são associados à marca na memória do consumidor (SPRY; PAPPU; CORNWELL, 2011). Keller (1998) refere que o brand equity proporciona um ativo estratégico que maximiza a desempenho da marca em longo-prazo, tornando a marca mais rentável por meio dos benefícios a ela associados, criando, assim, uma maior fidelidade, a possibilidade de cobrar um preço premium, melhores rácios de vendas etc. (MARTOS-PARDAL, 2012). Com o agravamento da situação econômica mundial, os salários a descer e o aumento do custo de vida, o consumidor opta por hábitos de consumo mais racionais e muda a sua consciência de valor (ANG, 2001), sendo que as marcas de distribuidor podem ser a alternativa mais econômica. O presente estudo tem como objetivos estudar o brand equity das marcas de distribuidor assim como identificar as variáveis que podem contribuir para a sua formação. Tem ainda como objetivos estudar os impactos do brand equity destas marcas na intenção de compra e no word-of-mouth (WOM). Nesta pesquisa, utilizaram-se antecedentes de duas naturezas distintas: um conjunto de variáveis ligadas à dimensão da marca (prestígio da marca, identidade da marca, valor percebido, confiança e reputação) e à sua estratégia de marketing, materializadas no seu marketing-mix e price deals; um outro conjunto de variáveis ligadas às atitudes e aos comportamentos do consumidor como sensibilidade ao preço, o comportamento hedônico e a identificação com a marca. Como consequentes do brand equity, apresentamos variáveis como a intenção de compra e o WOM (word-of-mouth).

Este trabalho encontra-se dividido em 5 partes fundamentais. A primeira parte diz respeito à apresentação do quadro conceptual e hipóte- ses de investigação. A segunda e a terceira parte dizem respeito, respetivamente, à metodologia, análise e discussão dos resultados. Finalmente, encontramos, na quarta parte, a discussão e as conclusões e, na quinta e última parte, as Limitações e Futuras Linhas de Investigação.

\section{QUADRO CONCEPTUAL E HIPÓ- TESES DE INVESTIGAÇÃO}

\subsection{QUADRO CONCEPTUAL}

As marcas do distribuidor apresentam hoje uma qualidade aceitável e estão cada vez mais a ser procuradas pelo consumidor. Isto se deve ao fato de seu custo ser bastante inferior ao custo dos produtos das marcas de fabricante, conseguindo satisfazer a mesma necessidade procurada. As marcas de distribuição estão a apostar mais na inovação e no design, de forma a poderem acompanhar a qualidade acrescentada pelas marcas de fabricante. A inovação é sempre uma mais-valia, pois acrescenta valor às marcas e só assim poderão comunicar a sua utilidade para o consumidor quer ao nível emocional, quer funcional, adotando estratégias de marketing eficazes para a imagem da marca em longo prazo (KELLER, 1998). O price deals e a sensibilidade aos preços serão variáveis de estudo, pois, com a situação econômica mundial, cada vez mais instável, o consumidor viu-se obrigado a optar por comprar produtos mais baratos, que satisfazem as mesmas necessidades dos mais caros, traduzindo-se, assim, um equilíbrio entre o útil e o agradável. Keller (1993) apresentou o brand equity baseado no cliente, centrando-se no conhecimento da marca, levando em consideração a conscientização da marca e a sua imagem, denominando-as de abordagens diretas e indiretas. Já Aaker (1996a) construiu o seu modelo de brand equity baseado em cinco dimensões: lealdade; brand awareness; a qualidade percebida; as associações e os ativos da marca. Quanto ao modelo de Yoo e Donthu (2001, p. 2), usado neste estudo, considera que brand equity "significa a medição do capital cognitivo e comportamental do valor da marca em nível do consumidor individual, através de um inquérito 
aos consumidores baseadas em experiências de consumo". O modelo concetual foi desenhado com o objetivo de estruturar o procedimento da investigação seguindo variáveis que vão ao encontro dos objetivos gerais deste artigo. $\mathrm{O}$ brand equity é o centro de toda a investigação, uma vez que se pretende saber se o consumidor tem uma atitude positiva em face das marcas de distribuidor e se realmente estas são possuidoras de valor para o consumidor. Assim, a forma mais correta e viável para investigar a situação acima mencionada foi dividir as varáveis em dois grupos. Em um grupo, consideram-se as variáveis relativas à marca (aquilo que a marca é para o consumidor) e a estratégia de marketing da empresa e no outro, as variáveis relativas ao consumidor (características que podem fazer diferença na atitude deste no momento da decisão de compra), vistas como antecedentes do brand equity. Também incidimos a nossa análise nos consequentes do brand equity. $\mathrm{Na}$ figura abaixo representada, podemos observar o modelo de investigação que será objeto de estudo deste trabalho.

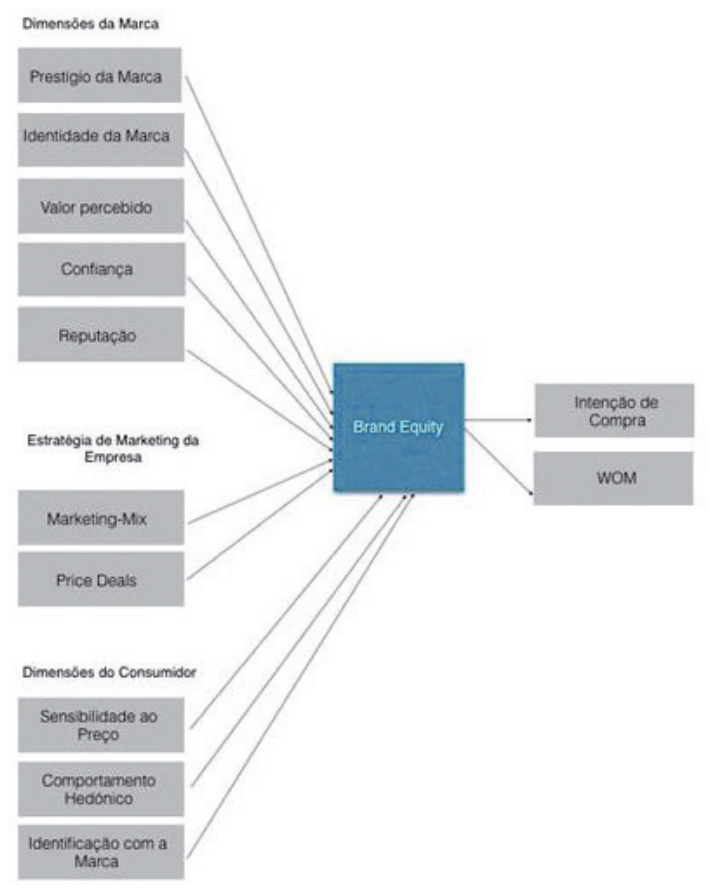

Figura 1 - Representação gráfica do modelo concetual da investigação- Brand equity e a atitude do consumidor perante as marcas da distribuição Fonte: elaboração própria.

\subsection{HIPÓTESES DE INVESTIGAÇÃO}

\subsubsection{Prestígio da Marca}

O prestígio da marca é definido como o grau com que os consumidores associam estatuto e estima a uma marca (BAEK; KING, 2011; STEENKAMP; BATRA; ALDEN, 2003; STOKBURGER; RATNESHWAR; SEN, 2012). Desta forma, os consumidores tenderão a ver marcas prestigiantes como sinal de status, riqueza ou poder (STEENKAMP; BATRA; ALDEN, 2003). Destaca-se ainda o fato de os consumidores, ao adquirirem uma marca de prestígio, esperarem que esta possa lhe possa fazer mostrar um maior desempenho e qualidade (GARFEIN, 1989; HEISEY, 1990; VIGNERON; JOHNSON, 1999; BAEK; KIM; YU, 2010). O prestígio da marca está por outro lado também ligado à qualidade percebida, à lealdade e ao conhecimento/associações da marca. $\mathrm{O}$ valor de uma marca para o consumidor está, desta forma, associado tanto ao prestígio como à qualidade percebida, bem como a outras variáveis que contribuem para a construção do brand equity, nas suas três dimensões (STEENKAMP; BATRA; ALDEN, 2003). Sendo uma marca detentora de tais características, isto leva a que, certamente, o consumidor a perceba como sendo uma marca de qualidade, o que fará que ela tenha clientes satisfeitos e com vontade de repetir a compra. Consequentemente, as marcas prestigiantes tornar-se-ão mais conhecidas. Desta forma, propomos as seguintes hipóteses:

H1a: O prestígio da marca tem um efeito positivo na qualidade percebida.

H1b: O prestígio da marca tem um efeito positivo na lealdade.

H1c: O prestígio da marca tem um efeito positivo no brand awareness.

\subsubsection{Identidade da Marca}

Segundo Kapferer (1991), a identidade é definida como um sentimento de existir enquanto indivíduo coerente e específico, que 
assume a sua história e tendo o seu lugar em relação aos outros. A identidade é o que diferencia a marca das outras, o que a torna única e especial para o consumidor, criando um valor para o cliente, sendo um elemento fulcral para a construção do brand equity (KAPFERER, 2000). Sendo uma marca caracterizada por sua diferenciação no mercado, isto levará certamente o consumidor a atribuir-lhe qualidade e a querer repetir a compra. Por outro lado, podemos certamente também afirmar que marcas com forte identidade serão marcas que ocupam um lugar de destaque no mercado, o que leva a crer que elas ganhem notoriedade. Assim se propõem as seguintes hipóteses:

H2a: A identidade da marca tem um efeito positivo na qualidade percebida.

H2b: A identidade da marca tem um efeito positivo na lealdade.

H2c: A identidade da marca tem um efeito positivo no brand awareness.

\subsubsection{Valor Percebido}

O valor percebido diz respeito a uma avaliação global que os consumidores fazem após ponderarem o que recebem de uma marca e aquilo que dão (ZEITHAML, 1988). Por outras palavras, o valor percebido consiste em uma avaliação da utilidade de uma determinada marca, sendo esta feita a partir de uma comparação entre aquilo que um consumidor recebe (e.g. qualidade, satisfação, etc.) e dá a uma determinada marca (e.g. tempo, dinheiro, esforço, etc.) em face daquilo que poderia dar e receber por parte de outra marca (NETEMEYER et al., 2004). Desta forma, o valor percebido corresponde à utilidade de um produto ou serviço, que, habitualmente, está relacionado com a perceção do consumidor, havendo uma troca dos benefícios por custos (NILSON, 1992; OSTROM; IACOBUCCI, 1995; JENSEN, 1996; WOODRUFF; GARDIAL, 1996; HESKETT; SASSER; SCHLESINGER, 1997). Um estudo realizado por Garretson, Fisher e Burton (2002) refere que o valor percebido interfere positivamente na compra tanto das marcas de distribuidor como nas marcas de fabricante. Desta forma, o valor percebido está ligado à qualidade percebida $\mathrm{e}$ também às associações à marca, podendo também contribuir para a satisfação do consumidor sendo uma das medidas mais importantes para o brand equity e futura atitude positiva na construção de lealdade. Assim se propõem as seguintes hipóteses:

H3a: O valor percebido tem um efeito positivo na qualidade percebida.

H3b: O valor percebido tem um efeito positivo na lealdade.

H3c: O valor percebido tem um efeito percebido no brand awareness.

\subsubsection{Confiança}

A confiança de marca está definida como a vontade de o consumidor comum confiar na habilidade da marca para executar a sua função (CHAUDHURI; HOLBROOK, 2002), contribuindo para a concretização de suas expectativas e intenções. A confiança na marca é assim baseada em uma promessa de valor para o consumidor. A confiança está assim relacionada com a predisposição de o consumidor confiar na marca para corresponder às suas expetativas (CHAUDHURI; HOLBROOK, 2002). A confiança é assim uma variável que ajuda à construção de lealdade do consumidor (CHAUDHURI; HOLBROOK, 2001). Ela permite que sejam criadas relações estáveis e duradouras, criando um valor notório da marca. A confiança pode ser um antecedente do brand equity porque cria relações que são fortemente estáveis (MORGAN; HUNT, 1994). Assim se propõem as seguintes hipóteses:

H4a: A confiança tem uma relação positiva com qualidade percebida.

H4b: A confiança tem uma relação positiva com a lealdade.

H4c: A confiança tem uma relação positiva com o brand awareness 


\subsubsection{Reputação}

Argenti e Druckenmiller (2004, p. 369) definem reputação como "uma representação coletiva de múltiplas imagens constituintes da empresa, construída ao longo do tempo e baseada em programas de identidade da empresa". Segundo De Chernatony e Harris (2000), a reputação é perceção da marca em longo prazo; é algo que torna a marca mais estável (ALMEIDA, 2014). A reputação pode estar relacionada a consequências que sejam favoráveis às marcas, mesmo em nível económico. Uma reputação corporativa favorável proporciona vantagem competitiva à marca e um maior valor (FOMBRUN; SHANLEY, 1990). Marcas reputadas são normalmente marca conhecidas do público em geral como sendo aquelas que vendem produtos de qualidade e com as quais os consumidores pretendem manter uma relação estável e duradoura. Assim se propõe a seguinte hipótese: H5a: A Reputação tem um efeito positivo na qualidade percebida.

H5b: A Reputação tem um efeito positivo na lealdade.

H5c: A Reputação tem um efeito positivo no brand awareness.

\subsubsection{Marketing-Mix}

O marketing-mix constituído pelos 4 P's (product, price, promotion, place) é o conjunto dos elementos da estratégia de marketing que a empresa controla (KOTLER; KELLER, 2009). O marketing-mix é uma variável que interfere fortemente nas perceções dos consumidores e tem um efeito positivo na construção do brand equity (qualidade percebida, lealdade $e$ brand awareness) (YOO; DONTHU, 2001). Marcas que trabalham de uma forma eficaz a diferentes componentes do marketing-mix irão certamente usufruir de benefícios vantajosos em nível de perceção da qualidade dos seus produtos, lealdade e brand awareness de suas próprias marcas. Assim se propõem as seguintes hipóteses:

H6a: O marketing-mix Price tem um efeito positivo na qualidade percebida.
H6b: O marketing-mix Price tem um efeito positivo na lealdade.

H6c: O marketing-mix Price tem um efeito positivo no brand awareness.

H7a: O marketing-mix Imagem de loja tem um efeito positivo na qualidade percebida.

H7b: O marketing-mix Imagem de loja tem um efeito positivo na lealdade.

H7c: O marketing-mix Imagem de loja tem um efeito positivo no brand awareness

H8a: O marketing-mix Gastos em publicidade tem um efeito positivo na qualidade percebida.

H8b: O marketing-mix Gastos em publicidade tem um efeito positivo na lealdade.

H8c: O marketing-mix Gastos em publicidade tem um efeito positivo no brand awareness

H9a: O marketing-mix Intensidade da distribuição tem um efeito positivo na qualidade percebida.

H9b: O marketing-mix Intensidade da distribuição tem um efeito positivo na lealdade.

H9c: O marketing-mix Intensidade da distribuição tem um efeito positivo no brand awareness.

\subsubsection{Price Deals}

O consumidor é propenso a responder positivamente a promoções a que está exposto, pois o preço que é apresentado sob a forma de promoção é sempre mais atrativo, influenciando-o, assim, na decisão de compra (LICHTENSTEIN; NETEMEYER; BURTON, 1990; LICHTENSTEIN; RIDGWAY; NETEMEYER, 1993). As promoções podem constituir valor para a marca se o consumidor for particularmente sensível aos preços. Esta variável é apresentada de forma independente do marketing mix uma vez que a intensidade de sua percepção supera, muitas vezes, o efeito das restantes políticas da empresa (SWANI; YOO, 2010). É uma variável que é habitualmente tratada de forma independente e que pode produzir resultados opostos aos das restantes políticas de marketing e com impacto direto no 
comportamento do consumidor (ANGEL; MANUEL, 2005). Estudos realizados por Monroe e Chapman (1987) e por Burton et al. (1998) referem que o preço de promoção faz que os consumidores aumentem a perceção de valor relativamente ao produto da marca em questão pelo que se espera que esta atitude se reflita em nível da qualidade percebida, lealdade e brand awareness. Assim se propõem as seguintes hipóteses:

H10a: A promoção de preços tem um feito positivo na qualidade percebida.

H10b: A promoção de preços tem um efeito positivo na lealdade.

H10c: A promoção de preços tem um efeito positivo no brand awareness.

\subsubsection{Sensibilidade ao Preço}

No estudo realizado por Sinha e Batra (1999), refere-se que os consumidores têm uma maior sensibilidade ao preço devido ao aumento da quota de mercado das marcas do distribuidor, e que o nível de sensibilidade ao preço na categoria do produto é uma variável explicativa na compra das marcas de distribuidor. $\mathrm{O}$ fato de um consumidor ser sensível ao preço pode fazer que este seja favoravelmente influenciado a comprar as marcas da distribuição e conferir-lhe maior valor (FREITAS, 2010). De acordo com Dhar e Hoch (1997), os consumidores mais sensíveis aos preços são aqueles que têm mais tendência para comprar produtos da marca do distribuidor.

Assim se propõem as seguintes hipóteses:

H11a: A sensibilidade ao preço tem um efeito positivo na qualidade percebida.

H11b: A sensibilidade ao preço tem um efeito positivo na lealdade.

H11c: A sensibilidade ao preço tem um efeito positivo no brand awareness.

\subsubsection{Comportamento Hedónico}

O hedonismo é definido por Schwartz e Rubel (2005) como um valor medido por itens como o prazer, a autoindulgência e uma vida com um lado mais prazeroso. O comportamento hedônico tem assim um efeito positivo no brand equity, pois, se o consumidor está disposto a comprar um produto pelo prazer ou experiência que lhe dá, a marca consegue ter um valor mais elevado. As marcas que proporcionarem tais sentimentos a seus consumidores serão certamente marcas que serão mais recordadas, marcas percecionadas como aquelas que têm produtos de qualidade e com as quais os consumidores pretendem manter uma relação duradoura. Assim se propõem as seguintes hipóteses:

H12a: O comportamento hedônico tem um efeito positivo na qualidade percebida.

H12b: O comportamento hedônico tem um efeito positivo na lealdade.

H12c: O comportamento hedônico tem um efeito positivo no brand awareness.

\subsubsection{Identificação com a Marca}

O consumidor, ao identificar-se com determinada marca, significa que terá uma afinidade ou amor à marca (TUSKEJ, GOLOB; PODNAR, 2013), assim como uma identificação de sua personalidade com a personalidade da marca. Identificando-se com uma determinada marca, o consumidor atribuir-lhe-á qualidade e quererá manter-se certamente próximo dela. Sendo um estatuto nem sempre fácil de ser alcançado, esta característica terá certamente também um impacto nos índices de recordação da marca detentora de tais características. Assim se propõem as seguintes hipóteses:

H13a: A identificação com a marca tem um efeito positivo na qualidade percebida.

H13b: A identificação com a marca tem um feito positivo na lealdade.

H13c: A identificação com a marca tem um feito positivo no brand awareness.

\subsubsection{Intenção de Compra}

A intenção de compra indica um comportamento futuro de um indivíduo perante uma determinada marca. Esta variá- 
vel tem sido muito importante para estudar o comportamento dos consumidores, pois é um indicador forte de uma possível compra no futuro. Segundo os autores Fishbein e Ajzen (1975) e Schiffman e Kanuk (2007), quando os consumidores apresentam uma intenção de compra positiva, têm à partida um compromisso de marca positivo. Yoo e Donthu (2001) sugerem que um brand equity mais elevado sugere a possibilidade de uma maior predisposição para comprar. Existem diversas opiniões sobre a intenção de compra. Alguns autores defendem que o preço é um grande decisor para a intenção de compra; outros, que o WOM (word-o$f$-mouth) ou as pessoas próximas também têm uma influência determinante (MATTEI; MACHADO; OLIVEIRA, 2006). Sendo a marca percecionada como sendo uma marca com valor, isto fará certamente que o consumidor esteja consciente que tomou a decisão certa no momento de compra pelo que quererá certamente repetir esse comportamento. Assim se propõem as seguintes hipóteses:

H14a: A qualidade percebida tem um impacto na intenção de compra.

H14b: A lealdade tem um impacto na intenção de compra.

H14c: O brand awareness tem um impacto na intenção de compra.

H15: A intenção de compra tem um efeito positivo no WOM (worth-of-mouth).

\subsubsection{WOM (word-of-mouth)}

Segundo Brown et al. (2005), o word-of-mouth ocorre quando a informação relativa a produtos, serviços, lojas, empresas etc. se espalha de uns consumidores para os outros. Esta pode ser vista como a resposta mais importante que pode surgir de um relacionamento entre consumidores e marcas (BROWN et al., 2005). Quando os consumidores percecionam determinada marca como sendo a única opção, dada a ligação emocional que o liga a esta, eles estarão dispostos a passar a palavra sobre essa marca simplesmente porque acreditam verdadeiramente nela (TUSKEJ; GOLOB; PODNAR, 2013). O word-of-mouth é algo que influencia bastante o comportamento do consumidor perante as marcas e as compras realizadas. Como é um meio de comunicação informal, torna-se mais facilmente viral, isto é, conversas e opiniões dadas pelo círculo social: de amigos, familiar ou profissional, faz que haja uma maior confiança na qualidade de determinado serviço ou produto da marca em questão, levando, assim, a uma maior disponibilidade para experimentar ou adquirir os produtos da marca. Segundo Arndt (1967), o "boca a boca" define um comportamento de comunicação verbal entre os consumidores sobre determinada marca, não apresentando qualquer intenção comercial (LIN; LU, 2010, p. 22). O WOM não tem que ser feito somente por meio do contato direto face-to-face, pode ser realizado através de outros meios, como por exemplo, internet, telefone, etc (BUTTLE, 1998). Assim propõe-se as seguintes hipóteses:

H16a: A qualidade percebida tem impacto no WOM (word-of-mouth).

H16b: A lealdade tem impacto no WOM (word-of-mouth).

H16c: O brand awareness tem um impacto no WOM (word-of-mouth).

\section{METODOLOGIA}

\subsection{RECOLHA DE DADOS}

No âmbito deste trabalho de investigação, realizou-se uma pesquisa de método quantitativo e de dedução de hipóteses para o recolhimento de dados. O método escolhido foi o método por questionário sendo colocado on-line por meio da plataforma Google Docs para que também, de uma forma rápida e eficaz, disponibilizasse as respostas devidamente codificadas e, assim sendo, 
possivelmente transcrevesse esta base para o software Statistical Package for the Social Sciences- SPSS. A terminologia das questões foi feita com base no formato de escolha múltipla, em uma escala de 1 (discordo totalmente) a 5 (concordo totalmente), em que os inquiridos apenas poderiam escolher uma opção que se ajustasse mais de acordo com aquilo que sentiam. O questionário realizado foi restrito apenas às perguntas que são colocadas; ou seja, não havia margem para o inquirido responder ao que não está diretamente explícito nas questões. Destaca-se também o fato de o consumidor escolher livremente a marca de distribuidor que tinha em mente quando respondeu ao inquérito e de se ter utilizado a metodologia snowball ou "bola-de-neve" para a seleção dos inquiridos. Ao longo deste estudo, os itens utilizados para medir as diferentes variáveis tiveram por base escalas traduzidas de vários trabalhos anteriormente apresentados, dos quais se destacam os seguintes: Baek, Kim e Yu (2010) (prestígio da marca); Bhattacharya e Sen (2003) (identidade da marca); Dodds, Monroe e Grewal (1991) e Aaker (1996b) (valor percebido); Taylor, Celuch e Goodwind (2004) e Chaudhuri e Holbrook (2001) (confiança); García de los Salmones, Crespo e Bosque (2005) (reputação); Yoo, Donthu e Lee (2000) (Marketing-mix); Yoo, Donthu e Lee, (2000) (Price deals); Lall e Bell (2003), Ayala e Neslin (2005), Demoulin e Zidda (2006) e Mueller (2007) (sensibilidade ao preço); Babin, Darden e Griffin (1994) (comportamento hedónico); Mael e Ashforth (1992), Stokburger, Ratneshwar e Sem (2012) (identificação com a marca) e Carroll e Ahuvia (2006) (intenção de compra e passa palavra positivo). Destaca-se, também, o fato de este trabalho de investigação ter contemplado a análise de 237 inquéritos validados e o recolhimento destes dados ter ocorrido ao longo dos meses de abril de maio de 2015. Relativamente aos dados recolhidos, importa destacar que a amostra foi composta por homens $(45,1 \%)$ e mulheres $(54,9 \%)$. Relativamente à idade, a maior parte dos inquiridos esteve entre os 24-29 anos, com 80 inquiridos. No que toca à escolaridade, o maior número de indivíduos respondeu que o seu grau educacional correspondia à licenciatura, 131 indivíduos $(55,3 \%)$, o que, de certa forma, vem justificar a faixa etária dos respondentes. Quanto à ocupação, 100 indivíduos responderam ser trabalhadores e estudantes, em simultâneo $(42,2 \%), 73$ eram estudantes $(30,8 \%)$, $20,7 \%$ eram apenas trabalhadores, $5,1 \%$ desempregados (as) e os restantes $1,3 \%$ reformados (as).

Por meio da análise fatorial confirmatória, e no que diz respeito à avaliação do modelo global, utilizando o software estatístico IBM SPSS AMOS (versão 21), verificou-se que o modelo apresentava um valor de ajustamento satisfatório. Podemos constatar que relativamente ao RMSEA (Root Mean Square Error of Approximation) e ao CFI (Comparative Fit Index), o modelo apresenta um ajustamento bom, 0,06 e 0,901 , respetivamente. Quanto ao TLI (Tucker-Lewis Index), apresentou um ajustamento aceitável segundo Marôco (2010) com 0,885 , o IFI apresenta o valor de 0,903 que corresponde a um bom ajustamento. Depois de uma análise detalhada do modelo de medidas (avaliação dos coeficientes estandardizados, normalidade multivariada, fiabilidade de cada indicador, fiabilidade de cada variável latente e análise da validade discriminante), verificamos que não existem valores que se afastem dos valores recomendados pela literatura. Para todas as variáveis latentes, quer os valores da fiabilidade compósita (CR) quer os valores da variância extraída média (AVE), estão, respetivamente, acima dos valores recomendados de 0.7 e 0.5. Após a análise destes indicadores, verificamos que todos os construtos utilizados no modelo conceptual respeitam os critérios estipulados por Hair et al. (1995). 
Tabela 1- Desvio Padrão; Diagonal a negrito - Alpha de Cronbach; C.R. - Fiabilidade compósita; AVE - Variância média extraída

\begin{tabular}{|c|c|c|c|c|c|c|c|c|c|c|c|c|c|c|c|c|c|c|c|}
\hline & DP & $x 1$ & $\times 2$ & $x 3$ & $x 4$ & $\times 5$ & $\times 6$ & $x 7$ & $x 8$ & $\times 9$ & $\times 10$ & $\times 12$ & $x 13$ & $\mathrm{x} 14$ & x15 & $x 16$ & $x 17$ & CR & AVE \\
\hline $\begin{array}{l}\text { Qualidade } \\
\text { Percebida }(x 1)\end{array}$ & 0,693 & 0,852 & & & & & & & & & & & & & & & & 0,860 & 0,673 \\
\hline Lealdade $(\times 2)$ & 0,622 & 0,272 & 0,872 & & & & & & & & & & & & & & & 0,872 & 0,695 \\
\hline $\begin{array}{l}\text { Brand A wareness } \\
\text { (x3) }\end{array}$ & 0,894 & 0,129 & 0,295 & 0,899 & & & & & & & & & & & & & & 0,900 & 0,749 \\
\hline $\begin{array}{l}\text { Prestígio da Marca } \\
\text { (x4) }\end{array}$ & 0,756 & 0,367 & 0,222 & 0,187 & 0,931 & & & & & & & & & & & & & 0,932 & 0,821 \\
\hline $\begin{array}{l}\text { Identidade da } \\
\text { Marca(x5) }\end{array}$ & 0,868 & 0,412 & 0,194 & 0,109 & 0,899 & 0,904 & & & & & & & & & & & & 0,906 & 0,762 \\
\hline $\begin{array}{l}\text { Marketing-Mix } \\
\text { Price }(x 6)\end{array}$ & 0,999 & 0,055 & 0,029 & 0,042 & 0,289 & 0,299 & 0,943 & & & & & & & & & & & 0,945 & 0,851 \\
\hline $\begin{array}{l}\text { M.M Imagem da } \\
\text { Loja }(x 7)\end{array}$ & 0,614 & 0,367 & 0,296 & 0,162 & 0,355 & 0,299 & 0,050 & 0,884 & & & & & & & & & & 0,850 & 0,719 \\
\hline $\begin{array}{l}\text { M.M Gastos em } \\
\text { Publicidade ( } x 8)\end{array}$ & 1,047 & 0,012 & 0,000 & 0,012 & 0,008 & 0,381 & 0,028 & 0,024 & 0,887 & & & & & & & & & 0,892 & 0,734 \\
\hline $\begin{array}{l}\text { M.M Intensidade da } \\
\text { Distribuição(x }(x)\end{array}$ & 0,806 & 0,023 & 0,030 & 0,085 & 0,036 & 0,009 & 0,002 & 0,009 & 0,187 & 0,826 & & & & & & & & 0,834 & 0,628 \\
\hline Reputação $(\times 10)$ & 0,473 & 0,278 & 0,122 & 0,135 & 0,130 & 0,021 & 0,036 & 0,139 & 0,105 & 0,143 & 0,817 & & & & & & & 0,825 & 0,613 \\
\hline Price Deals $(\times 11)$ & 0,746 & 0,027 & 0,026 & 0,004 & 0,044 & 0,131 & 0,206 & 0,002 & 0,054 & 0,008 & 0,078 & 0,920 & & & & & & 0,924 & 0,802 \\
\hline $\begin{array}{l}\text { Sensibilidade ao } \\
\text { Preço }(x 12)\end{array}$ & 0,515 & 0,023 & 0,039 & 0,000 & 0,000 & 0,031 & 0,010 & 0,007 & 0,016 & 0,009 & 0,035 & 0,034 & 0,821 & & & & & 0,829 & 0,619 \\
\hline $\begin{array}{l}\text { Comportamento } \\
\text { Hedónico }(x 13)\end{array}$ & 0,945 & 0,018 & 0,038 & 0,133 & 0,000 & 0,007 & 0,002 & 0,000 & 0,015 & 0,050 & 0,011 & 0,017 & 0,000 & 0,874 & & & & 0,877 & 0,706 \\
\hline $\begin{array}{l}\text { Identificação coma } \\
\text { Marca (x14) }\end{array}$ & 0,686 & 0,030 & 0,194 & 0,637 & 0,093 & 0,000 & 0,036 & 0,076 & 0,003 & 0,159 & 0,075 & 0,001 & 0,003 & 0,176 & 0,831 & & & 0,845 & 0,649 \\
\hline $\begin{array}{l}\text { Confiança na Marca } \\
\text { (x15) }\end{array}$ & 0,676 & 0,044 & 0,362 & 0,308 & 0,063 & 0,043 & 0,010 & 0,399 & 0,004 & 0,002 & 0,145 & 0,033 & 0,000 & 0,004 & 0,079 & 0,774 & & 0,938 & 0,790 \\
\hline $\begin{array}{l}\text { Intenção de compra } \\
\text { (x16) }\end{array}$ & 0,669 & 0,179 & 0,121 & 0,436 & 0,094 & 0,035 & 0,003 & 0,047 & 0,005 & 0,134 & 0,174 & 0,004 & 0,022 & 0,199 & 0,321 & 0,042 & 0,893 & 0,784 & 0,548 \\
\hline $\begin{array}{l}\text { WOM(Worth-of- } \\
\text { Mouth) }(\times 17)\end{array}$ & 0,882 & 0,364 & 0,264 & 0,224 & 0,167 & 0,060 & 0,002 & 0,181 & 0,004 & 0,017 & 0,158 & 0,024 & 0,002 & 0,056 & 0,343 & 0,280 & 0,228 & 0,895 & 0,739 \\
\hline
\end{tabular}

Fonte: elaboração própria.

\section{ANÁLISE E DISCUSSÃO DE RESULTADOS}

\subsection{RESULTADOS}

A tabela 2 apresenta os resultados do modelo estrutural que testa as hipóteses propostas para esta investigação. A tabela apresenta os coeficientes estandardizados e o nível de significância que permitem estabelecer as hipóteses que são efetivamente corroboradas.

Tabela 2- Resultado do Teste de Hipóteses

\begin{tabular}{|c|c|c|c|}
\hline Variável & Hipótese & SRW & $\mathbf{P}$ \\
\hline $\mathrm{PM} \rightarrow \mathrm{QP}$ & H1a & $-0,046$ & $>0,05$ \\
\hline $\mathrm{PM} \rightarrow$ Lealdade & $\mathrm{H} 1 \mathrm{~b}$ & 0,239 & $>0,05$ \\
\hline $\mathrm{PM} \rightarrow \mathrm{BA}$ & H1c & 0,359 & $>0,05$ \\
\hline IDENTIMARCA $\rightarrow$ QP & $\mathrm{H} 2 \mathrm{a}$ & 0,456 & $>0,05$ \\
\hline IDENTIMARCA $\rightarrow$ Lealdade & $\mathrm{H} 2 \mathrm{~b}$ & $-0,111$ & $>0,05$ \\
\hline IDENTIMARCA $\rightarrow$ BA & $\mathrm{H} 2 \mathrm{c}$ & $-0,272$ & $>0,05$ \\
\hline $\mathrm{CM} \rightarrow \mathrm{QP}$ & $\mathrm{H} 4 \mathrm{a}$ & 0,0337 & ** \\
\hline $\mathrm{CM} \rightarrow$ Lealdade & $\mathrm{H} 4 \mathrm{~b}$ & 0,332 & $* *$ \\
\hline $\mathrm{CM} \rightarrow \mathrm{BA}$ & $\mathrm{H} 4 \mathrm{c}$ & 0,169 & ** \\
\hline REPUTAÇÃO $\rightarrow \mathrm{QP}$ & H5a & 0,203 & * \\
\hline REPUTAÇÃO $\rightarrow$ Lealdade & $\mathrm{H} 5 \mathrm{~b}$ & $-0,028$ & $>0,05$ \\
\hline REPUTAÇÃO $\rightarrow$ BA & $\mathrm{H} 5 \mathrm{c}$ & 0,176 & $* *$ \\
\hline $\mathrm{MMP} \rightarrow \mathrm{QP}$ & H6a & 0,03 & $>0,05$ \\
\hline
\end{tabular}




\begin{tabular}{|c|c|c|c|}
\hline MMP $\rightarrow$ Lealdade & H6b & 0,051 & $>0,05$ \\
\hline $\mathrm{MMP} \rightarrow \mathrm{BA}$ & H6c & $-0,081$ & $>0,05$ \\
\hline $\mathrm{MMIL} \rightarrow \mathrm{QP}$ & $\mathrm{H} 7 \mathrm{a}$ & 0,101 & $>0,05$ \\
\hline MMIL $\rightarrow$ Lealdade & $\mathrm{H} 7 \mathrm{~b}$ & 0,159 & $>0,05$ \\
\hline $\mathrm{MMIL} \rightarrow \mathrm{BA}$ & $\mathrm{H} 7 \mathrm{c}$ & 0,053 & $>0,05$ \\
\hline $\mathrm{MMGP} \rightarrow \mathrm{QP}$ & H8a & $-0,0085$ & $>0,05$ \\
\hline MMGP $\rightarrow$ Lealdade & $\mathrm{H} 8 \mathrm{~b}$ & $-0,091$ & $>0,05$ \\
\hline $\mathrm{MMGP} \rightarrow \mathrm{BA}$ & $\mathrm{H} 8 \mathrm{c}$ & $-0,132$ & $*$ \\
\hline $\mathrm{MMID} \rightarrow \mathrm{QP}$ & H9a & 0,063 & $>0,05$ \\
\hline MMID $\rightarrow$ Lealdade & $\mathrm{H} 9 \mathrm{~b}$ & 0 & $>0,05$ \\
\hline $\mathrm{MMID} \rightarrow \mathrm{BA}$ & H9c & $-0,013$ & $>0,05$ \\
\hline $\mathrm{PD} \rightarrow \mathrm{QP}$ & H10a & 0,119 & * \\
\hline $\mathrm{PD} \rightarrow$ Lealdade & $\mathrm{H} 710 \mathrm{~b}$ & 0,158 & $*$ \\
\hline $\mathrm{PD} \rightarrow \mathrm{BA}$ & H10c & $-0,0117$ & * \\
\hline $\mathrm{SP} \rightarrow \mathrm{QP}$ & H11a & 0,039 & $>0,05$ \\
\hline $\mathrm{SP} \rightarrow$ Lealdade & H11b & 0,213 & ** \\
\hline $\mathrm{SP} \rightarrow \mathrm{BA}$ & H11c & $-0,0117$ & $>0,05$ \\
\hline $\mathrm{CH} \rightarrow \mathrm{QP}$ & H12a & 0,117 & $*$ \\
\hline $\mathrm{CH} \rightarrow$ Lealdade & $\mathrm{H} 12 \mathrm{~b}$ & 0,009 & $>0,05$ \\
\hline $\mathrm{CH} \rightarrow \mathrm{BA}$ & $\mathrm{H} 12 \mathrm{c}$ & 0,085 & $>0,05$ \\
\hline IDENTIFICMARCA $\rightarrow$ QP & H13a & $-0,159$ & $* *$ \\
\hline IDENTIFICMARCA $\rightarrow$ Lealdade & $\mathrm{H} 13 \mathrm{~b}$ & 0,288 & $* *$ \\
\hline IDENTIFICMARCA $\rightarrow$ BA & H13c & 0,628 & $* *$ \\
\hline $\mathrm{QP} \rightarrow \mathrm{IC}$ & H14a & 0,014 & $>0,05$ \\
\hline Lealdade $\rightarrow$ IC & $\mathrm{H} 14 \mathrm{~b}$ & $-0,033$ & $>0,05$ \\
\hline $\mathrm{BA} \rightarrow \mathrm{IC}$ & $\mathrm{H} 14 \mathrm{c}$ & 0,602 & $* *$ \\
\hline $\mathrm{IC} \rightarrow \mathrm{WOM}$ & H15 & 0,209 & $* *$ \\
\hline $\mathrm{QP} \rightarrow \mathrm{WOM}$ & H16a & 0,392 & $* *$ \\
\hline Lealdade $\rightarrow$ WOM & H16b & 0,202 & $* *$ \\
\hline $\mathrm{BA} \rightarrow \mathrm{WOM}$ & H16c & 0,129 & $* *$ \\
\hline
\end{tabular}

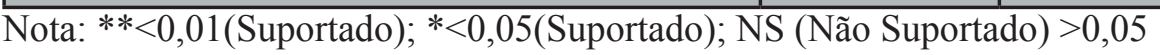

Fonte: elaboração própria.

Como podemos averiguar, contrariamente ao inicialmente suposto quando à definição da hipótese H1a, o prestígio da marca não tem um impacto na qualidade percebida, $(b=$ $-0,046, p>0,05)$. Na relação entre o prestígio da marca e a lealdade (H1b), também aqui não é verificado qualquer tipo de relação entre as variáveis $(b=0,239, p>0,05)$. Na hipótese $H 1 c$, consta-se, mais uma vez, que não há uma rela- ção significativa entre as variáveis prestígio da marca e o brand awareness $(b=0,359, p>0,05)$. Aparentemente, o prestígio não parece afetar o comportamento dos consumidores. De fato, a relação qualidade preço costuma aparecer como determinante, pois, apesar da notoriedade crescente, ainda não parecem ser marcas que se destaquem por seu prestígio. No caso da relação entre a variável da identidade da marca e 
a qualidade percebida (H2a), verifica-se que a relação entre as variáveis nesta hipótese não é suportada, $(b=0,456, p>0,05)$. O mesmo acontece no que diz respeito à relação entre a identidade da marca e a lealdade (H2b), ( $b=-0,111$, $\mathrm{p}>0,05)$. No que diz respeito à relação entre a variável da identidade da marca e o brand awareness (H2c), deparamo-nos com a mesma situação da hipótese referida anteriormente $(b=-0,272, p>0,05)$. Pelas mesmas razões, as marcas de distribuidor parecem não ter ganhado ainda uma identidade que as diferencie positivamente das marcas premium. No que diz respeito à $\mathrm{H} 4 \mathrm{a}$, verificamos, tal como o inicialmente proposto, a existência de uma relação positiva entre a confiança na qualidade percebida $(b=0,337, p<0,01)$. Na relação entre a confiança da marca e a lealdade (H4b), confirma-se igualmente um impacto significativo entre as variáveis $(b=0,332, p<0,01)$. No caso da relação entre as variáveis confiança na marca e o brand awareness $(\mathrm{H} 4 \mathrm{c})$, verifica-se a existência de um impacto positivo como inicialmente era esperado, $(b=0.169, p<0,01)$. Na hipótese H5a, respeitante à relação entre a reputação e a qualidade percebida, foi constatado um impacto positivo na relação entre estas variáveis $(b=0,203$, $\mathrm{p}<0,05)$; contudo na relação entre a reputação e a lealdade (H5b), não foi confirmada uma relação positiva $(b=-0,028, p>0,05)$. No entanto, na hipótese $\mathrm{H} 5 \mathrm{c}$ relativa à relação existente entre a reputação e o brand awareness, confirma-se o que, inicialmente, era esperado: existe assim um impacto da reputação sobre o brand awareness $(\mathrm{b}=0,176, \mathrm{p}<0,01)$. Na relação entre o marketing-mix price e a qualidade percebida (H6a), não se verifica uma relação significativa entre elas $(b=0,030, p>0,05)$. No caso da relação entre o marketing-mix price e a lealdade, respeitante à hipótese H6b, também não há qualquer grau de significância, e a hipótese não é suportada, $(b=0,051, p>0,05)$. No caso da hipótese H6c, também não corroboramos a relação existente entre o marketing-mix price e o brand awareness, $(\mathrm{b}=-0,081, \mathrm{p}>0,05)$. No que diz respeito à relação entre a variável de marketing-mix imagem de loja e a qualidade percebida (H7a), verificamos que ela não apresenta um efeito estatisticamente significativo $(b=0,101, p>0,05)$. O mesmo fato se verifica para a hipótese $H 7 b(b=0,159, p>0,05)$, tal como na relação entre o marketing-mix imagem de loja e o brand awareness $(\mathrm{H} 7 \mathrm{c}),(\mathrm{b}=0,053$, $\mathrm{p}>0,05)$. Quanto à variável de marketing-mix gastos em publicidade, verificamos que ela não tem impacto na variável qualidade percebida $(b=-0,085, p>0,05)(H 8 a)$. A mesma situação se vem a verificar para a hipótese H8b, hipótese que estabelecia a relação entre o marketing-mix gastos em publicidade e a lealdade à marca $(b=-0,091, p>0,05)$. Na relação entre a variável marketing-mix gastos em publicidade e brand awareness, existe impacto que, no entanto, é negativo $(b=-0,132, p<0,05)$, alterando, assim, o pressuposto da H8c. No caso da hipótese H9a, hipótese que estabelecia uma relação positiva entre a variável de marketing-mix intensidade da distribuição e a qualidade percebida (H9a), verificamos que esta não foi corroborada pelos dados $(b=0,063, p>0,05)$. A mesma situação verificou-se na hipótese H9b, hipótese que considerava a relação positiva entre o marketing-mix intensidade da distribuição e a lealdade à marca $(\mathrm{H} 9 \mathrm{~b}),(\mathrm{b}=0,000, \mathrm{p}>0,05)$. Na hipótese H9c, hipótese que estabelecia a relação entre o marketing-mix intensidade da distribuição e o brand awareness, também o nosso estudo não conseguiu corroborar a hipótese inicialmente formulada $(b=-0,013, p>0,05)$. $\mathrm{Na}$ verdade, os esforços de marketing das marcas de distribuidor tendem a concentrar-se nos efeitos preço e, por esta razão, as restantes variáveis apresentam uma visibilidade reduzida e um impacto estatisticamente não significativo no valor das marcas.

Relativamente à relação existente entre o price deals e a qualidade percebida (H10a), verifica-se que, realmente, há um impacto positivo $(b=0,119, p<0,05)$. Tal fato se confirma na relação entre o price deals e a lealdade (H10b), $(b=0.158, p<0,05)$. No entanto, na hipótese $\mathrm{H} 10 \mathrm{c}$, verificou-se que o inicialmente suposto não se vem a confirmar, o price deals tem impacto no brand awareness, contu- 
do negativo, $(b=-0,0117, p<0,05)$. No caso da relação entre a sensibilidade ao preço e a qualidade percebida (H11a), também não se conseguiu confirmar o que foi inicialmente suposto, sendo que esta variável não tem um efeito significativo na qualidade percebida $(b=0,039$, $\mathrm{p}>0,05)$. Na hipótese H11b, verificou-se que a sensibilidade ao preço tem um impacto positivo na lealdade $(b=0,213, p<0,01)$. No entanto, o mesmo fato não ocorre com a relação entre a variável de sensibilidade ao preço e o brand awareness $(\mathrm{H} 11 \mathrm{c}),(\mathrm{b}=0,044, \mathrm{p}>0,05)$. Na verdade, a sensibilidade ao preço deverá apresentar uma relação mais significativa com a intenção de compra do que com o valor das marcas, com um efeito que parece supor uma menor relevância desta variável, quando se fala de marcas de distribuidor. No que diz respeito à relação entre o comportamento hedônico e a qualidade percebida (H12a), verificou-se, tal como o inicialmente suposto, uma relação positiva entre ambas as variáveis $(b=0,117$, $\mathrm{p}<0,05)$. Quanto à relação entre o comportamento hedônico e a lealdade, não ficou aqui demonstrada uma relação significativa entre estas variáveis $(\mathrm{H} 12 \mathrm{~b}),(\mathrm{b}=0,009, \mathrm{p}>0,05)$. Isso aconteceu com a hipótese $\mathrm{H} 12 \mathrm{c}$, ou seja, o comportamento hedônico não tem impacto no brand awareness $(b=0,085, p>0,05)$. Como se pode verificar, contrariamente ao inicialmente suposto, a identificação com a marca tem um impacto na qualidade percebida; no entanto, esse impacto é negativo $(b=-0,159, p<0,05)$, o que não suporta a hipótese H13a. Relativamente à relação entre a variável identificação com a marca e a lealdade, o impacto existente é positivo $(b=0.288, p<0,01)$; ou seja, confirmado o que anteriormente foi proposto, que a identificação com a marca tem um efeito positivo na lealdade à marca, suportando, assim, a hipótese H13b. No que respeita à hipótese $\mathrm{H} 13 \mathrm{c}$, mais uma vez como foi inicialmente suposto, a identificação com a marca apresenta um impacto positivo no brand awareness $(b=0,628, p<0,01)$. Analisando agora o impacto dos constituintes do brand equity (qualidade percebida, lealdade e brand awareness), nos consequentes do brand equity, verificou-se que a qualidade percebida não tem impacto na intenção de compra ( $b=0,014, p>0,05)$, não suportando a hipótese H14a. No que diz respeito ao impacto da lealdade sobre a intenção de compra (H14b), verificou-se que não conseguimos suportar a hipótese inicialmente proposta $(b=-0,033, p>0,05)$. Já no caso da hipótese $\mathrm{H} 14 \mathrm{c}$ relativa ao impacto do brand awareness sobre a intenção de compra, confirmou-se a existência de um impacto positivo $(b=0,602, p<0,01)$. Finalmente, também foi analisada a relação entre a intenção de compra e WOM (H15) e verificou-se, tal como o inicialmente formulado, a existência de uma relação positiva entre ambas as variáveis consideradas $(b=0,209, p<0,01)$. Na relação entre a qualidade percebida e o WOM (H16a), verificou-se existir um efeito positivo $(b=0,392$, $\mathrm{p}<0,01)$, tal como na hipótese $\mathrm{H} 16 \mathrm{~b}$, relação entre a lealdade e o WOM $(b=0,202, p<0,01)$. $\mathrm{Na}$ relação entre brand awareness e o WOM (H16c) $(b=0,129, p<0,01)$, verifica-se que a hipótese encontra suporte nestes resultados.

\section{DISCUSSÃO E CONCLUSÕES}

Esta pesquisa traz um conhecimento acrescido para o desenvolvimento do comportamento do consumidor em face do valor das marcas do distribuidor. Ao passo que os estudos anteriores se caracterizavam em determinantes específicos do brand equity; este abrange variáveis como o marketing-mix (YOO; DONTHU; LEE, 2000), nas variáveis individuais a sensibilidade ao preço (ALMEIDA, 2014), o comportamento hedônico (BABIN; DARDEN; GRIFFIN, 1994) e a identificação com a marca (MAEL; ASHFORTH, 1992; STOKBURGER; RATNESHWAR; SEN, 2012) e ainda as atitudes em face das marcas. Este estudo é de natureza mais holística e engloba variáveis, quer de natureza individual, quer as atitudes em face das marcas, quer, finalmente, a estratégia de marketing da empresa. Destacou-se o fato de outras investigações futuras poderem apro- 
fundar, de forma transcendente, o impacto destas variáveis nas marcas do distribuidor. Todavia e desde logo, este estudo apresentou uma natureza mais global e envolveu várias categorias de antecedentes, o que marca uma diferença perante a investigação passada. Em seguida, e não menos importante, constatou-se que a variável central do modelo, o brand equity, é um denominador que interpreta e avalia o valor da marca da forma mais abrangente. Nesta investigação, o brand equity foi estudado por meio do modelo de Yoo e Donthu (2001), que tem vindo a apresentar resultados satisfatórios, sendo constituído pela qualidade percebida, lealdade e associações à marca. A presente investigação tinha como objetivo central estudar o brand equity das marcas de distribuidor, conhecer as variáveis que mais contribuíam para a sua formação bem como analisar algumas de suas consequências. Após a análise e utilização da modelagem SEM, que relaciona as variáveis em estudo com as variáveis latentes, conseguiu-se concluir que todos os testes apresentam os valores necessários para serem considerados bons.

\subsection{IMPLICAÇÕES AO NÍVEL ACADÊMICO E PRÁTICO}

Esta investigação apresenta um conjunto de implicações quer em nível acadêmico quer em prático. Desde logo, ela combina os efeitos relativos às variáveis do marketing-mix com as variáveis relativas ao consumidor, como antecedentes do brand equity, em um único modelo, o que é inovador em face da investigação produzida nesta área. Do mesmo modo, o Brand Equity surge ao mediar as relações entre essas variáveis e a intenção de compra e o WOM, o que é verdadeiramente significativo e inovador, já que permite ir monitorando a força das marcas de distribuidor na mente dos clientes e em seu comportamento. Esta combinação de variáveis em um único modelo, testada por recurso à modelagem de equações estruturais, significa também um progresso, dada a dimensão do modelo, que, ainda assim, obteve um FIT muito aceitável, permitindo extrair conclusões simultâneas em diferentes ângulos.

Depois de efetuados os testes de hipóteses, concluiu-se que alguns antecedentes do brand equity não tinham impacto positivo sobre ele, principalmente nas variáveis relativas à marca e à estratégia de marketing da empresa. A reputação, a confiança na marca e a promoção dos preços foram as variáveis que mostraram mais significância em seus impactos, o que vai ao encontro do definido anteriormente por vários autores (CHAUDHURI; HOLBROOK, 2001; MORGAN; HUNT, 1994; FOMBRUN; SHANLEY, 1990; MONROE; CHAPMAN, 1987; BURTON et al., 1998). Quanto às variáveis individuais do consumidor, a identificação com a marca apresentou um impacto positivo na lealdade e no brand awareness, mas negativo na qualidade percebida. $\mathrm{Na}$ variável do comportamento hedônico, foi apenas verificada uma relação positiva entre esta variável e a qualidade percebida; já na sensibilidade ao preço, essa relação apenas se constatou na lealdade, o que é consistente com o anteriormente apresentado por Freitas (2010). Já nos consequentes do brand equity, a intenção de compra teve apenas uma relação positiva com o brand awareness, a qualidade percebida, lealdade e brand awareness (brand equity) impactam de forma positiva no WOM. Também foi testada a relação entre os dois consequentes de brand equity que resultou em uma relação positiva. Levando em consideração o panorama atual ao nível econômico, é essencial que as marcas conheçam os seus consumidores, principalmente as marcas de distribuição, que em face da crise, são as mais procuradas devido ao fato de seus produtos serem relativamente inferiores às marcas de fabricante.

\section{LIMITAÇÕES E FUTURAS LINHAS DE INVESTIGAÇÃO}

Uma das limitações é que este método não se generaliza a todos os consumidores, pois o questionário é apenas uma situação 
esporádica, ou seja, o inquirido responde consoante o que está a sentir naquele exato momento, bem como não tem a oportunidade de justificar certas questões visto que elas são colocadas de forma numérica, podendo levar a uma interpretação não esclarecida do que o inquirido realmente sente em relação à marca.

Outra ideia das limitações do artigo pode estar associada às escalas utilizadas. Embora fossem obtidas por meio de escalas já validadas pela literatura, algumas poderiam ser ajustadas de forma a obter uma relação mais significativa do comportamento do consumidor perante o valor das marcas de distribuição. Como sugestões para futuras investigações, propõe-se uma pesquisa dentro do mesmo objetivo principal do modelo conceitual desta investigação, incidindo, assim, nos dois tipos de produtos que o consumidor pode adquirir das marcas do distribuidor, um produto com um lado funcional e outro produto ligado às emoções, um produto hedônico. Nesta investigação, a variável comportamento hedônico apenas apresentou significância com a qualidade percebida, mas tendo uma visão geral de todos os resultados; pode-se observar que houve dados positivos para a identificação com a marca, tal como na confiança do consumidor para com a marca. Assim, se as marcas de distribuidor souberem qual o tipo de produto em questão que mais tem saída no mercado, poderão trabalhar estratégias específicas de forma a aumentar a relação com o hedonismo. Se esta situação for possível, poderá influenciar o prestígio e a identidade da marca positivamente. Sugere-se que se realize o mesmo estudo, com as mesmas escalas de medição, porém conseguindo uma amostra mais numerosa e um nível de faixa etária mais abrangente, pois, na amostra desta investigação, a maioria era jovem estudante, que, por vezes, ainda não possui experiência suficiente no ato de fazer compras.

\section{BRAND EQUITY: THE CUSTOMER BEHAVIOR TOWARDS RETAIL BRANDS}

\section{ABSTRACT}

Brand equity is not only relevant for the management of brands, but it can also offer a broad set of advantages to customers. The central goal of this research consists of investigating the variables that contribute most to the brand equity formation and, at the same time, to study the impact of brand equity on purchase intention and on word-of-mouth (WOM). A survey with a quantitative nature was conducted through a structured questionnaire, based on a sample of 237 respondents over 18 years of both sexes, using a digital data collection. The statistical analysis reveals that the variables price deals, identification with the brand and trust showed an overall positive impact on variables related to the construction of brand equity: perceived quality, loyalty and brand awareness. These dimensions of brand equity seem to have a positive impact on word-of-mouth, besides that the brand awareness dimension has a positive impact on purchase intent. Finally, the intention to purchase has a positive impact on WOM.

Keywords: Brand Equity. Private Labels. Costumer Behavior.

\section{REFERÊNCIAS}

AAKER, D. A. Measuring Brand Equity across products and markets. California Management Review, United States, v. 38, n. 3, p. 102-120, 1996a.

AAKER, D. A. Building Strong Brands. New York: The Free Press, 1996 b.

ALMEIDA, N. M. F. O comportamento do consumidor face às marcas de distribuidor em período de crise. 2014. 161 f. Dissertação (Mestrado em Gestão) - Faculdade de Economia da Universidade de Coimbra, Coimbra, 2014. 
ANG, S. H. Personality influences on consumption: insights from the asian economic crisis. Journal of International Consumer Marketing, United States, v. 13, n. 1, p. 5-21, 2001.

ANGEL, F.; MANUEL, J. The impact of marketing communication and price promotion on brand equity. Brand Management, United Kingdom, v. 12, n. 6, p. 431-44, 2005.

ARGENTI, P.; DRUCKENMILLER, B. Reputation and the Corporate Brand. Corporate Reputation Review, United Kingdom, v. 6, n. 4, p. 368-373, 2004.

ARNDT, J. Role of product-related conversations in the diffusion of a new product. Journal of Marketing Research, United States, v. 4, n 3, p. 291-295, 1967.

AYALA, G.; NESLIN, S. The current and future sales impact of a retail frequency retail program. Journal of Retailing, United Kingdom, v. 81, n. 4, p. 293-305, 2005.

BABIN, B. J.; DARDEN, W. R.; GRIFFIN, M. Work and/or fun: measuring hedonic and utilitarian shopping value. Journal of Consumer Research, United States, v. 20, p. 644-656, Mar. 1994.

BAEK, T. H.; KIM, J.; YU, J. H. The differential Roles of Brand Credibility and Brand Prestige in Consumer Brand Choice. Psychology \& Marketing, United States, v. 27, n. 7, p. 662678, 2010.

BAEK, T. H.; KING, K. W. Exploring the consequences of brand credibility in services. Journal of Services Marketing, United Kingdom, v. 25, n. 4, p. 260-272, 2011.

BALTAS, G. Determinants of store brand choice: a behavioral analysis. Journal of Product and Brand Management, United Kingdom, v. 6, n. 5, p. 315-324, 1997.
BHATTACHARYA, C. B.; SEN, S. Consumer-company identification: A framework for understanding consumers' relationships with companies. Journal of Marketing, United States, v. 67, n. 2, p. 76-88, 2003.

BINNINGER, A. S. Exploring the relationships between retail brands and consumer store loyalty. International Journal of Retail \& Distribution Management, United Kingdom, v. 36, n. 2, p. 94-110, 2008.

BROWN, T. J. et al. Spreading the word: investigating antecedents of consumers' positive word-of-mouth intentions and behaviors in a retail- ing context. Journal of the Academy of Marketing Science, United States, v. 33, n. 2, p. 123-138, 2005.

BURTON, S. et al. A scale for measuring attitude toward private label products and an examination of its psychological and behavioral correlates. Journal of the Academy of Marketing Science, United States, v. 26, n. 4, p. 293-306, 1998.

BUTTLE, F. A. Word-of-mouth: understanding and managing referral marketing. Journal of Strategic Marketing, United Kingdom, v .6, n. 3, p. 241-54, 1998.

CARroll, B. A.; AHUVIA, A. C. Some Antecedents And Outcomes Of Brand Love. Marketing Letters, United States, v. 17, n. 2, p. 79-89, 2006.

CHAUDHURI, A.; HOLBROOK, M. B. Product-class effects on brand commitment and Brand outcomes: The role of brand trust and brand affect. Journal of Brand Management, United Kingdom, v. 10, n. 1, p. 33-58, 2002.

CHAUDHURI, A.; HOLBROOK, M. B. The chain of effects from brand trust and brand affect to brand performance: the role of brand loyalty. Journal of Marketing, United States, v. 65 , p. 81-93, 2001. 
DE CHERNATONY, L ; HARRIS, F. Developing Corporate Brands Through Considering Internal and External Stakeholders. Corporate Reputation Review, United Kingdom, v. 3, n. 3, p 268-274, 2000.

DEMOULIN, N.; ZIDDA, P. On the differences between loyalty card adopters and nonadopters: the case of a new loyalty program in the grocery retail market. In: EMAC CONFERENCE, 35th., 2006, Greece. Proceedings... Greece: Athens Universi- ty of Economics and Business, 2006.

DHAR, S. K.; HOCH, S. Why store brand penetration varies by retailer: abstract. Marketing Science, United States, v. 16, n. 3, p. 208-227, 1997.

DICK, A.; JAIN, A.; RICHARDSON, P. Correlates of Stores Brand Proneness: Some Empirical Observations. Journal of Product and Brand Management, United Kingdom, v. 4, n. 4, p. 15-22, 1995.

DODDS, W. B.; MONROE, K. B.; GREWAL, D. Effects of price, brand, and store information on buyer's product evaluations. Journal of Marketing Research, United States, v. 28, p. 307-319, 1991.

FARQUHAR, P. H. Managing Brand Equity. Marketing Research, United States, v. 1, p. 24-33, 1989.

FISHBEIN, M.; AJZEN, I. Belief, attitude, intention, and behavior: an introduction to theory and research. Reading: Addison-Wesley, 1978.

FOMBRUN, C. J.; SHANLEY, M. What's in a name? Reputation-building and corporate strategy. Academy of Management Journal, United States, v. 33, p. 233-258, 1990.

FREITAS, D. S. R. Lealdade dos consumidores às marcas de distribuidor. 2010. $130 \mathrm{f}$. Dissertação (Mestrado em Gestão Comercial) - Faculdade de Economia- U.P, Porto, 2010.
GARCÍA DE LOS SALMONES, M.; CRESPO, H.; BOSQUE, I. Influence of corporate social responsibility on loyalty and valuation of services. Journal of Business Ethics, Netherlands, v. 61, p. 369-385, 2005.

GARFEIN, R. T. Cross-cultural perspectives on the dynamics of presti-ge. Journal of Services Marketing, United Kingdom, v. 3, p. 1724, 1989.

GARRETSON, J. A.; FISHER, D.; BURTON, S. Antecedents of private label attitude and national brand promotion attitude: similarities and differences. Journal of Retailing, United Kingdom, v. 78, n. 2, p. 91-99, 2002.

HAIR, J. F. et al. Multivariate Data Analysis with readings. 4th ed. New Jersey: Prentice-Hall, 1995.

HEISEY, F. L. Perceived quality and predicted price: use of the mini- mum information environment in evaluating ready-to-wear. Clothing and Textiles Research Journal, United States, v. 8, p. 22-8, 1990.

HESKETT, J. L.; SASSER, W. E.; SCHLESINGER, L. A. The service profit chain: how leading companies link profit and growth to loyalty, satisfaction, and value. New York: The Free Press, 1997.

JENSEN, H. R. The interrelationship between customer and consumer value. Asia Pacific Advances in Consumer Research, v. 2, p. 6063, 1996.

KAPFERER, J. N. Les marques, capital de l'entreprise. France: Les Éditions d'organisation, 1991.

KAPFERER, J. N. Strategic brand management. Creating powerful brands in consumer service and industrial markets. London: Kogan Page, 2000. 
KELLER, K. L. Strategic brand management: building, measuring, and managing brand equity. 2th ed. Upper Saddle River: Prentice Hall, 2003.

KELLER, K. L. Strategic brand management. New Jersey: Prentice-Hall, 1998.

KELLER, K. L. Conceptualizing, measuring, and managing customer-based brand equity. Journal of Marketing, United States, v. 57, p. 1-22, Jan. 1993.

KOTLER, P.; KELLER, K. L. Administração de marketing: a bíblia do marketing. São Paulo, Pearson, 2009.

LALL, R.; BELL, D. The impact of frequent shopper programs in grocery retailing. Quantitative Marketing and Economics, Netherlands, v. 1, n. 2, p. 179-202, 2003.

LAMEY, L. et al. How business cycles contribute to private-label success: evidence from the United States and Europe. Journal of Marketing, United States, v. 71, n. 1, p. 1-15, 2007.

LICHTENSTEIN, D. R.; NETEMEYER, R. G.; BURTON, S. Distinguishing coupon proneness from value consciousness: an acquisition-transaction utility theory perspective. Journal of Marketing, United States, v. 54, p. 54-67, July 1990.

LICHTENSTEIN, D. R.; RIDGWAY, N. M.; NETEMEYER, R. G. Price percep- tions and consumer shopping behaviour: a field study. Journal of Marketing Research, United States, v. 20, p. 234-245, May 1993.

LIN, Long-Yi; LU, Ching-Yuh. The influences of corporate image, rela- tionship marketing, and trust on purchase intention: the moderating effects of word-of- mouth. Tourism Review, United Kingdom, v. 65, n. 3, p. 16-34, 2010.
MAEL, F.; ASHFORTH, B. E. Alumni and their alma mater: A partial test of the reformulated model of organizational identification. Journal of Organizational Behavior, United Kingdom, v. 13, n. 2, p. 103-123, 1992.

MARÔCO, J. Análise de equações estruturais: fundamentos teóricos, software e aplicações. Pêro Pinheiro: ReportNumber, 2010.

MARTOS-PARDAL, M. Innovation and the market share of private labels. Journal of Marketing Management, United Kingdom, v. 28, n. 5-6, p. 695-715, May 2012.

MATTEI, D.; MACHADO, M.; OLIVEIRA, P. Comportamento do consumidor: fatores que influenciam no processo de decisão de compra dos consumidores finais. Revista de Ciências Empresariais, Maringá, v. 3, n. 2, p. 27-37, 2006.

MONROE, K. B.; CHAPMAN, J. D. Framing effects on buyers' subjective product evaluations. Advances in Consumer Research, United States, v. 14, p. 193-197, 1987.

MORGAN, R.; HUNT, S. The commitment-trust theory of relationship marketing. Journal of Marketing, United States, v. 58, n. 3, p. 20-38, 1994.

MUELLER, S. Loyalty programs and customer loyalty: a panel data analysis. In: EMAC CONFERENCE, 36th., 2007, Reykjavik. Proceedings... Reykjavik: EMC, 2007.

NETEMEYER, R. G. et al. Developing and validating measures of facets of customer-based brand equity. Journal of Business Research, United States, v. 57, n. 12, p. 209-224, 2004.

NILSON, T. H. Value-added marketing: marketing management for superior results. Berkshire: McGraw-Hill, 1992. 
OSTROM, A.; IACOBUCCI, D. Consumer trade-offs and the evaluation of services. Journal of Marketing, United States, v. 59, p. 1728, 1995.

SCHIFFMAN, L. G.; KANUK, L. L. Consumer behavior: its origins and strategic applications, consumer behavior. 9th ed. New Jersey: Pearson, 2007.

SCHWARTZ, S. H.; RUBEL, T. Sex differences in value priorities: cross-cultural and multimethod studies. Journal of Personality and Social Psychology, United States, v. 89, n. 6, p. 1010-1028, 2005.

SINHA, I.; BATRA, R. The effect of consumer price consciousness on private label purchase. International Journal of Research in Marketing, Netherlands, v. 16, n. 3, p. 237-251, 1999.

SPRY, Amanda; PAPPU, Ravi; CORNWELL, T. Bettina. Celebrity endorsement, brand credibility and brand equity. European Journal of Marketing, United Kingdom, v. 45, n.6, p. 882-909, 2011.

STEENKAMP, J. B. E. M.; BATRA, R.; ALDEN, D. L. How perceived brand globalness creates brand value. Journal of International Business Studies, United Kingdom, v. 34, p. 53-65, 2003.

STOKBURGER, S.; RATNESHWAR, N. S.; SEN, S. Drivers of consumer-brand identification. International Journal of Research in Marketing, Netherlands, v. 29, p. 406-418, 2012.

SWANI, K.; YOO, B. Pricing strategy \& practice Interactions between price and price deal. Journal of Product \& Brand Management, United Kingdom, v. 19, n. 2, p. 143-152, 2010.

TAYLOR, S.; CELUCH, K.; GOODWIND, S. The importance of Brand Equity to consumer loyalty. Journal of Product and Brand Management, United Kingdom, v. 13, n. 4, p. 217227, 2004.
TUSKEJ, U.; GOLOB, U.; PODNAR, K. The role of consumer-brand identification in building brand relationships. Journal of Business Research, United States, v. 66, n. 1, p. 53-59, 2013.

VIGNERON, F.; JOHNSON, L. W. A review and a conceptual frame-work for prestigeseeking consumer behavior. Academy of Marketing Science Review, v. 1, p. 1-15, 1999.

WOODRUFF, R. B.; GARDIAL, S. F. Know your customer: new approaches to understanding customer value and satisfaction. Cambridge: Blackwell Publications, 1996.

YOO, B.; DONTHU, N. Developing and validating a multidimensional consumer-based brand equity scale. Journal of Business Research, United States, v. 52, n. 1, p. 1-14, 2001.

YOO, B.; DONTHU, N.; LEE, N. An examination of selected marketing mix elements and brand equity. Academy of Marketing Science, United States, v. 28, n. 2, p 195-211, 2000.

ZEITHAML, V. A. Consumer perceptions of price, quality and value: a means end model and synthesis of evidence. Journal of Marketing, United States, v. 52, n. 3, p. 2-22, 1988. 